\title{
Short-term effects of oestradiol, T3 or insulin infusions on plasma concentrations and estimated hepatic balances of metabolites in energy-deprived preruminant calves
}

\author{
Y Chilliard 1, C Audigier 1, D Durand 2, S Auboiron 2, D Bauchart 2 \\ 1 INRA, laboratoire Sous-Nutrition des ruminants, Theix; \\ 2 INRA, laboratoire Croissance et Métabolismes des herbivores, Theix, \\ 63122 Saint-Genès-Champanelle, France
}

Effects of hormone infusions on intermediary and hepatic metabolism were studied in 4 preruminant male calves fed milk replacers according to energy and protein requirements, except during 3 experimental periods (d 18-27, 32-41 and 46-55 after birth) where energy allowance was only $45 \%$ of total requirements. Animals were fitted with catheters in the mesenteric artery, the portal vein and one hepatic vein at $8 \mathrm{~d}$ of age.

After $5 \mathrm{~d}$ of energy restriction during each experimental period, each animal received successively and alternately 3 hormonal treatments (T): $17 \beta$-oestradiol $(0,20 \mu \mathrm{g} / \mathrm{kg} \mathrm{BW})$, triodothyronin (T3, $30 \mu \mathrm{g} / \mathrm{kg} \mathrm{BW}$ ) or insulin (I, $0.72 \mathrm{IU} / \mathrm{kg} \mathrm{BW}$ ), infused during the first $h$ after feeding. Metabolite concentrations were determined in the hepatic vein at $0.5,1,2,3,5$ and $7 \mathrm{~h}$ after morning feeding, and in the mesenteric artery and the portal vein at $7 \mathrm{~h}$ to calculate hepatic balance using blood flow estimates, according to body weight and previous equations (Durand et al, 1988).

OT significantly decreased triglyceride concentrations at 2, 3, 5 and $7 \mathrm{~h}$ after feeding. T3T significantly decreased glucose $(2,3,5$ h) and triglyceride $(2,3,5,7 \mathrm{~h})$ concentrations. IT significantly decreased glucose $(0.5,1,2,3,5 \mathrm{~h})$ and $3-\mathrm{OH}-\mathrm{B}(0.5,3 \mathrm{~h})$, and increased NEFA $(1,2$ h) concentrations. Effects of hormone infusions on estimated hepatic balances were non significant, suggesting that changes in metabolite concentrations were due to short-term effects on liver metabolism or due to effects on extrahepatic tissues, if it is assumed that treatments did not affect hepatic blood flow.

Durand D, Bauchart D, Lefaivre J, Donnat JP (1988) J Dairy Sci 71, 1632-1637

Table I. Short-term effects of hormone infusions on hepatic metabolism.

\begin{tabular}{|c|c|c|c|c|c|c|c|}
\hline \multirow[t]{2}{*}{ Metabolite $(\mathrm{n}=4)$} & \multirow[t]{2}{*}{ Time ${ }^{1}$} & \multicolumn{2}{|c|}{$17 \beta$-Oestradiol } & \multicolumn{2}{|c|}{ Triiodothyronin } & \multicolumn{2}{|c|}{ Insulin } \\
\hline & & Control 2 & Treated & Control & Treated & Control & Treated \\
\hline \multicolumn{8}{|c|}{ Hepatic vein concentration } \\
\hline Glucose $(\mathrm{mg} / \mathrm{ml})$ & 2 & 1.00 & 0.96 & 0.95 & $0.77^{\star \star}$ & 1.00 & $0.48^{\star \star}$ \\
\hline Triglycerides $(\mathrm{mg} / \mathrm{ml})$ & 3 & 0.48 & $0.31^{\star \star}$ & 0.59 & $0.27^{\star \star}$ & 0.47 & 0.41 \\
\hline$N E F{ }^{3}(\mathrm{mM})$ & 2 & 0.26 & 0.32 & 0.30 & 0.29 & 0.23 & $0.82^{*}$ \\
\hline $3-\mathrm{OH}-\mathrm{B}^{4}(\mathrm{mM})$ & 3 & 0.17 & 0.20 & 0.15 & 0.15 & 0.18 & $0.13^{*}$ \\
\hline Urea $(\mathrm{mg} / \mathrm{ml})$ & 2 & 0.40 & 0.40 & 0.39 & 0.51 & 0.42 & 0.42 \\
\hline \multicolumn{8}{|c|}{ Estimated hepatic balance ${ }^{5}$} \\
\hline Glucose 6 & 7 & 1.4 & 0.6 & 3.2 & 2.7 & 1.3 & 1.4 \\
\hline Triglycerides 6 & 7 & -1.7 & -2.7 & -2.1 & -1.5 & -1.6 & -1.3 \\
\hline NEFA 7 & 7 & -6.4 & -5.1 & $-0.1^{8}$ & -0.1 & 0 & 4.8 \\
\hline $3-\mathrm{OH}-\mathrm{B}^{7}$ & 7 & 0.9 & 2.3 & 2.6 & 2.4 & 3.0 & 1.9 \\
\hline Urea 6 & 7 & 0.2 & 0.6 & 0.7 & 0.5 & 0.6 & 0.3 \\
\hline
\end{tabular}

\footnotetext{
1 Hours after feeding and after the beginning of hormone infusion; 2 the day before hormone infusion; ${ }^{3}$ nonesterified fatty acids; ${ }^{4}$ 3-hydroxy-butyrate; ${ }^{5}$ apparent production, or $(-)$ captation; ${ }^{6} \mathrm{mg} / \mathrm{min} / \mathrm{kg} \mathrm{BW} ;{ }^{7} \mathrm{micromol} / \mathrm{min} /$ kg BW; ${ }^{8} 3$ animals only; ", ** $=P<0.13,0.07$, respectively.
} 